\title{
Connexin 43 interacts with Bax to regulate apoptosis of pancreatic cancer through a gap junction-independent pathway
}

\author{
YAN SUN ${ }^{1,3^{*}}$, XIAOPING ZHAO ${ }^{2 *}$, YUTING YAO $^{2}$, XINYI QI $^{2}$, YAOZONG YUAN $^{3}$ and YU HU ${ }^{1}$ \\ ${ }^{1}$ Department of Geriatrics, Zhongshan Hospital, Fudan University, Shanghai 200032; \\ ${ }^{2}$ Department of Biochemistry and Molecular Biology, Ninth People's Hospital, Shanghai JiaoTong University \\ School of Medicine, Shanghai 200011; ${ }^{3}$ Department of Gastroenterology, Ruijin Hospital, \\ Shanghai JiaoTong University School of Medicine, Shanghai 200025, P.R. China
}

Received November 27, 2011; Accepted April 24, 2012

DOI: $10.3892 /$ ijo.2012.1524

\begin{abstract}
Connexins play important roles in many physiological and pathological processes. Although connexins are considered as tumor suppressors in several types of cancer, their roles in pancreatic cancer are unknown. In this study, we found that connexin 43 (Cx43) caused apparent apoptosis and growth inhibition in pancreatic cancer cells. The tumor-suppressive role of Cx43 was independent of the canonical gap junction pathway. In the context of apoptosis, $\mathrm{Cx} 43$ translocated to the mitochondria, where it interacted with Bax to initiate the mitochondrial apoptotic pathway. Following further examination of the $\mathrm{Cx} 43$ protein, we found that the 241-382aa region of $\mathrm{Cx} 43$ was required for interaction with Bax. Furthermore, this region was responsible for permeabilizing mitochondrial membrane potential. The results from the present study elucidate a novel mechanism of the $\mathrm{Cx} 43$-mediated regulation of apoptosis in pancreatic cancer.
\end{abstract}

\section{Introduction}

Pancreatic cancer is the fourth most frequent cause of cancer related mortality, with a 5-year overall survival of less than

Correspondence to: Professor Yu Hu, Department of Geriatrics, Zhongshan Hospital, Fudan University, 180 Fenglin Road, Shanghai 200032, P.R. China

E-mail: hu.yu@zs-hospital.sh.cn

Professor Yaozong Yuan, Department of Gastroenterology, Ruijin Hospital, Shanghai JiaoTong University School of Medicine, 197 2nd Ruijin Road, Shanghai 200025, P.R. China

E-mail: yyz28@medmail.com.cn

${ }^{*}$ Contributed equally

Abbreviations: Cx43, connexin 43; GJ, gap junction; $\beta$-GA, 18 - $\beta$-glycyrrhetinic acid; MMP, mitochondrial membrane potential; Cyt c, cytochrome c

Key words: pancreatic cancer, gap junction, connexin 43, Bax, apoptosis
5\%. In the United States, approximately 42,470 individuals are diagnosed with this condition and 35,240 die from the disease each year (1). To date, tumor resection has remained the only curative therapy for pancreatic carcinoma. Irradiation and chemotherapy do not have a significant therapeutic effect on pancreatic cancer (2). Therefore, pancreatic cancer still represents a therapeutic challenge in oncology.

Connexins (Cxs) are encoded by a multigene family; to date, 21 different $\mathrm{Cx}$ genes have been identified. Gap junctions (GJs), which are composed of $\mathrm{Cx}$ proteins, allow the direct exchange of small molecules between adjacent cells (3). A previous study showed that Cxs exert their function through a GJ-independent pathway. The underlying mechanisms of a GJ-independent pathway mainly involve protein interactions (4). The dysregulation of $\mathrm{Cx}$ expression has been associated with carcinogenesis of the lung, breast, prostate, liver, stomach and colon. Cxs are involved in the regulation of tumor proliferation, apoptosis, invasion and metastasis (5). Connexin 43 is the major isoform in the pancreas, yet few studies have addressed its role in pancreatic cancer (6). We performed this study in order to unravel the mechanism of the $\mathrm{Cx} 43$-regulated apoptosis of pancreatic cancer.

\section{Materials and methods}

Cell culture, plasmids and materials. Human pancreatic cancer cells (BxPC-3, SW1990, PaTu8988, PANC-1, AsPC-1 and CFPAC-1) were grown in high glucose DMEM or RPMI-1640, supplemented with $10 \%(\mathrm{v} / \mathrm{v}) \mathrm{FBS}$ at $37^{\circ} \mathrm{C}$ with $5 \% \mathrm{CO}_{2}$. The siRNAs against $\mathrm{Cx} 43$ oligonucleotides were synthesized by Ambion (Grand Island, NY, USA) with the sequence 5'-GAUGAUAACCAGAA UUCTA-3'. Non-silencing control siRNA was synthesized using scrambled sequence as the negative control (NC). The dominant-negative $\mathrm{Cx} 43$ mutant (Cx43N) was constructed by deletion of amino acids 130-136 from the cytoplasmic loop. This mutant is effective as a dominant-negative inhibitor of GJs. The transfection of siRNA or plasmids was applied using Lipofectamine 2000 (Invitrogen, Carlsbad,CA,USA) according to the manufacturer's instructions.

Assay for GJ intercellular communication. The GJ assay protocol was employed based on a modification of the methods 

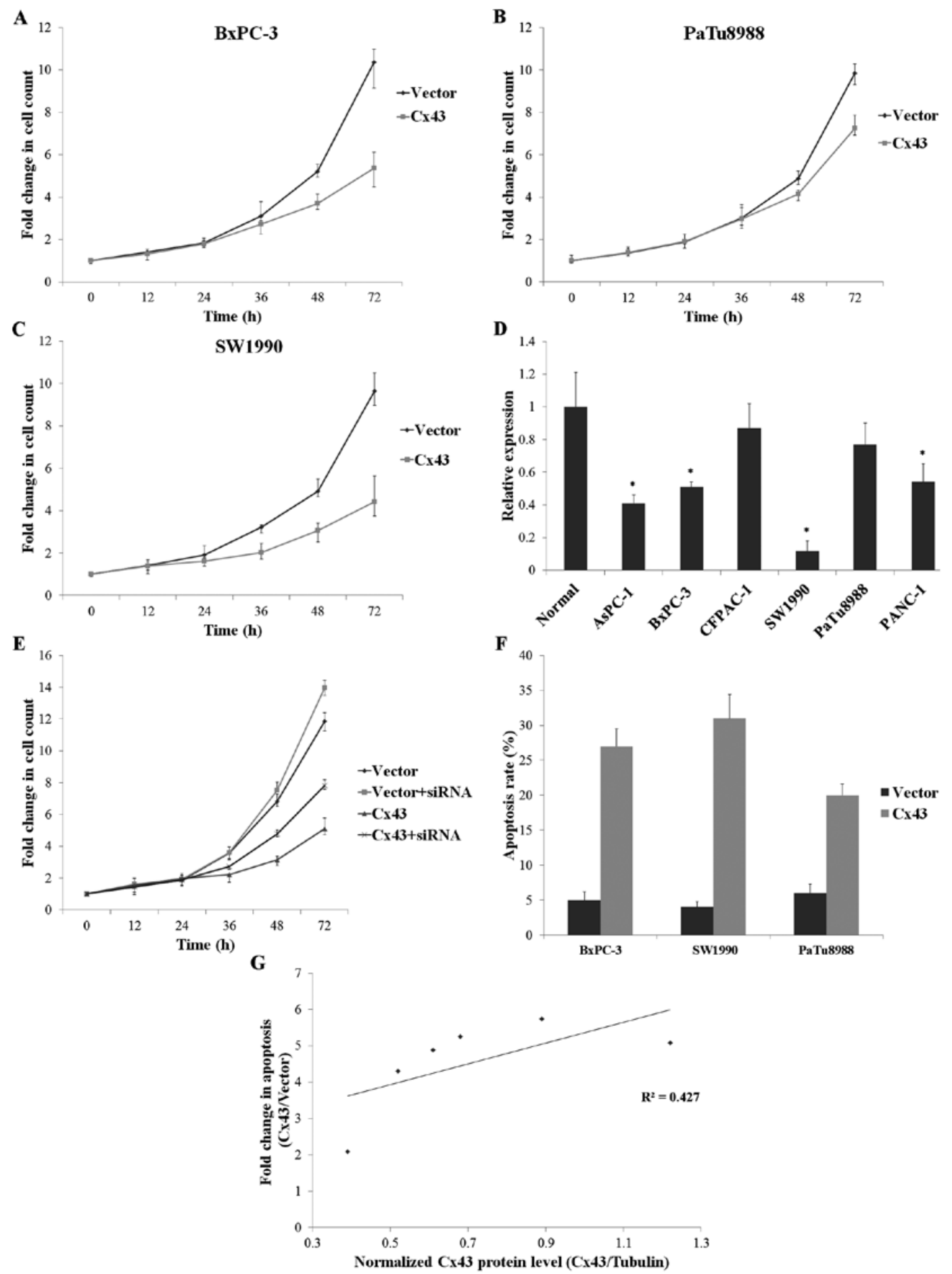

Figure 1. Cx43 inhibits the growth of human pancreatic cancer cells. (A-C) Cell proliferation was analyzed in BxPC-3, PaTu8988 and SW1990 cells. Cells were transfected with $\mathrm{Cx} 43$ or the control plasmid. Cell numbers were recorded for 3 days. (D) Analysis of relative Cx43 expression in the pancreatic cancer cell lines, AsPC-1, BxPC-3, CFPAC-1, SW1990, PaTu8988 and PANC-1. (E) Cell proliferation was analyzed in BxPC-3 cells with Cx43 plasmid or siRNA. (F) Apoptotic rate was assessed in BxPC-3, SW1990 and PaTu8988 cells by Annexin V/PI assay. (G) The ordinate shows changes in apoptotic rate. The abscissa was normalized to $\mathrm{Cx} 43$ protein levels. Dots represent the average changes in AsPC-1, BxPC-3, CFPAC-1, SW1990, PaTu8988 and PANC-1 cells. Data represent the means \pm SEM values from at least 3 separate experiments.

described by Goldberg et al (7). Donor and recipient cell populations were differentially labeled for 30 min with the GJ-permeable dye, calcein AM, and the lipophilic dye, DiIC $_{18}$ (Molecular Probes, Eugene, OR, USA), in DMEM containing $10 \%$ FBS, respectively. After washing, donor cells were trypsinized and added to acceptor cells at a 1:5 (donor: acceptor) ratio for $3 \mathrm{~h}$ at $37^{\circ} \mathrm{C}$. Co-cultures were harvested and subjected to FACS analysis. If GJ intercellular communication (GJIC) occurred, calcein was transferred from donor cells (green) to recipient cells (red). Thus, double-labeled cells represented the communicating cells in this assay. Cytometric data were expressed in terms of the coupling index (coupled acceptor cells/total potential acceptor cells) per donor cell and were plotted as the means \pm SEM of 3 experiments. 
A
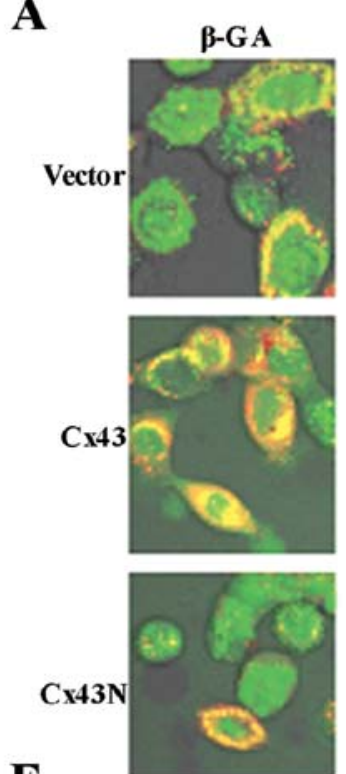

$\mathbf{E}$

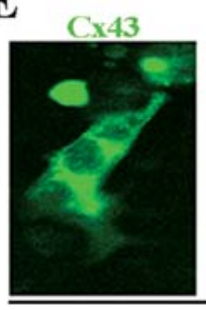

Mito

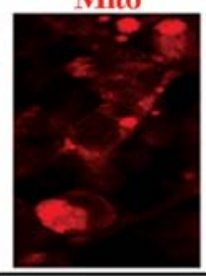

Control

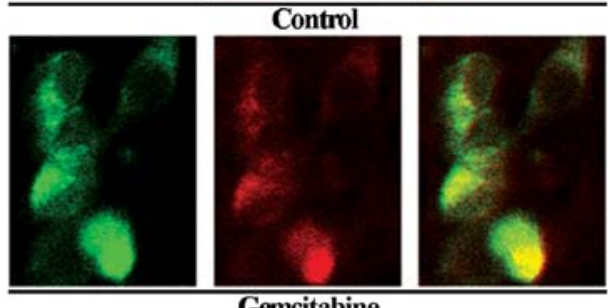

Gemcitabine

Overiay

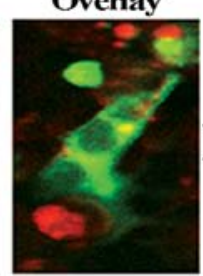

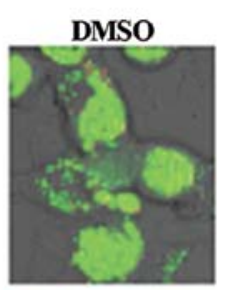
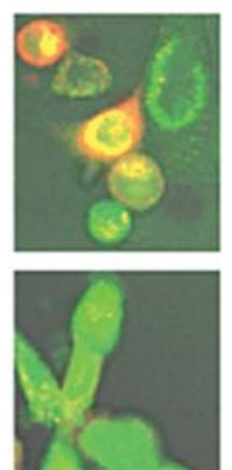

B

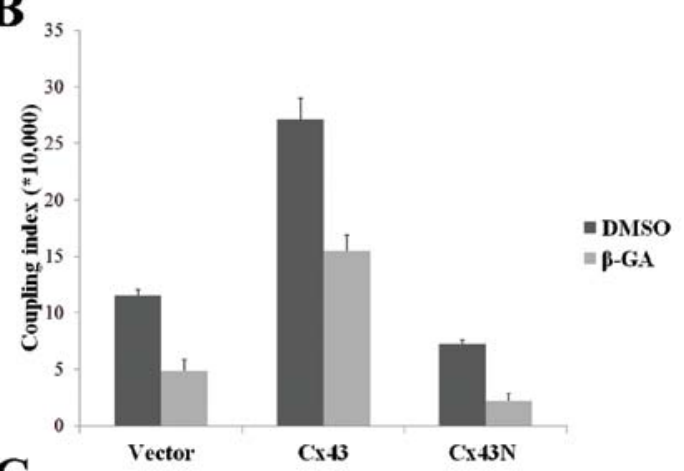

C

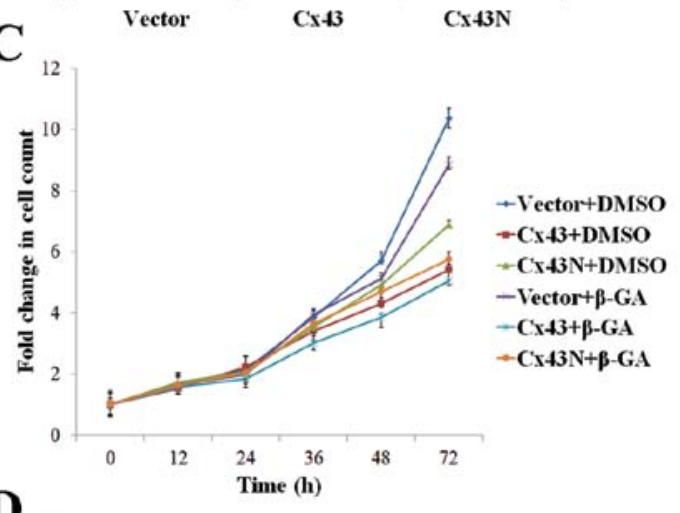

D

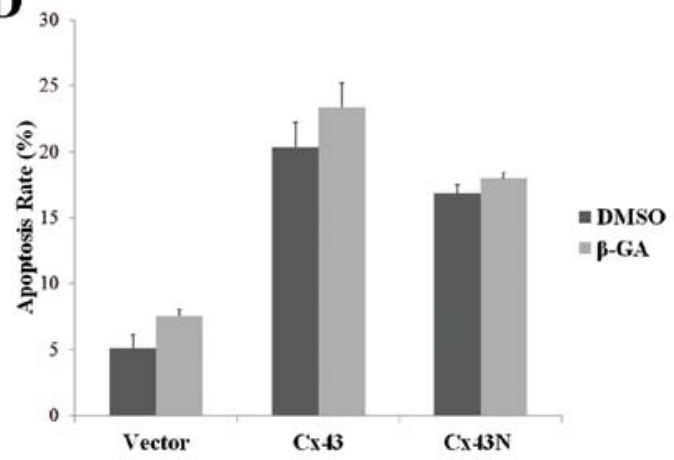

Figure 2. The tumor-suppressive role of $\mathrm{Cx} 43$ is independent of GJ. (A) GJs were analyzed by confocal microscopy in cells transfected with $\mathrm{Cx} 43$, Cx43N or control plasmid. Calcein was transferred from donor cells (green) to recipient cells (red). Double-labeled cells (yellow) represent the communicating cells. (B) Coupling index was calculated according to the ratio of communicating cells per recipient cells. After cells were transfected with $\mathrm{Cx} 43$, Cx43N or control plasmid, they were further treated with or without $\beta$-GA. The apoptotic rate (C) and growth curve (D) were also analyzed in these cells. (E) Cx43 (green) subcellular localization was analyzed by confocal microscopy in BxPC-3 cells with or without gemcitabine. Mitochondria were labeled by MitoTracker dye (red). The overlay images (yellow) reveal the co-localization of $\mathrm{Cx} 43$ with the mitochondria. Data represent the means \pm SEM values from at least 3 separate experiments.

Analysis of mitochondrial depolarization. JC-1 (Invitrogen) was employed to measure mitochondrial depolarization in BxPC-3 cells. Mitochondrial depolarization was indicated by a decrease in the red/green fluorescence intensity ratio. Cells were incubated with $5 \mu \mathrm{g} / \mathrm{ml}$ of JC- 1 at $37^{\circ} \mathrm{C}$ for $15 \mathrm{~min}$ and then analyzed by fluorescence-activated cell sorter.

Co-immunoprecipitation. For immunoprecipitation, $200 \mu \mathrm{g}$ of cell lysate were incubated with $25 \mu \mathrm{l}$ each of agarose A and $\mathrm{G}$ (Invitrogen) in $500 \mu \mathrm{l}$ total volumes for $1 \mathrm{~h}$. Immunoprecipitating antibody was added with an additional $3 \mathrm{~h}$ of incubation at $4^{\circ} \mathrm{C}$ with constant rotation. The complex was washed 3 times. It was then resuspended in SDS loading buffer. After boiling, the supernatant was loaded for western blot analysis. The total protein in the input lysate was approximately $1 / 10$ of the amount used for immunoprecipitation. The antibodies used were: $\mathrm{Cx} 43$ (Sigma, St. Louis, MO, USA), Bax, Bcl-2 and GAPDH (Cell Signaling Technology, Danvers, MA, USA).
Statistical analysis. All experiments were performed in triplicate and the results are expressed as the means \pm SEM. The data were analyzed with the Student's t-test or by one-way analysis of variance (ANOVA) and $\mathrm{P} \leq 0.05$ denoted a statistically significant difference.

\section{Results}

Cx43 induces apoptosis of pancreatic cancer cells. We examined the proliferation rates in pancreatic cancer cells transfected with $\mathrm{Cx} 43$. Cell growth was significantly inhibited in the presence of $\mathrm{Cx} 43$. Of note, growth inhibition was more apparent in SW1990 and BxPC-3 cells, but not in PaTu8988 cells (Fig. 1A-C). The possible reason was that PaTu8988 cells had a relatively higher level of $\mathrm{Cx} 43$ (Fig. 1D). We then tried to use siRNA to rescue the growth inhibition induced by $\mathrm{Cx} 43$. Growth inhibition was recovered in the cells co-transfected with DNA and Cx43 siRNA. The Cx43 knockdown promoted 


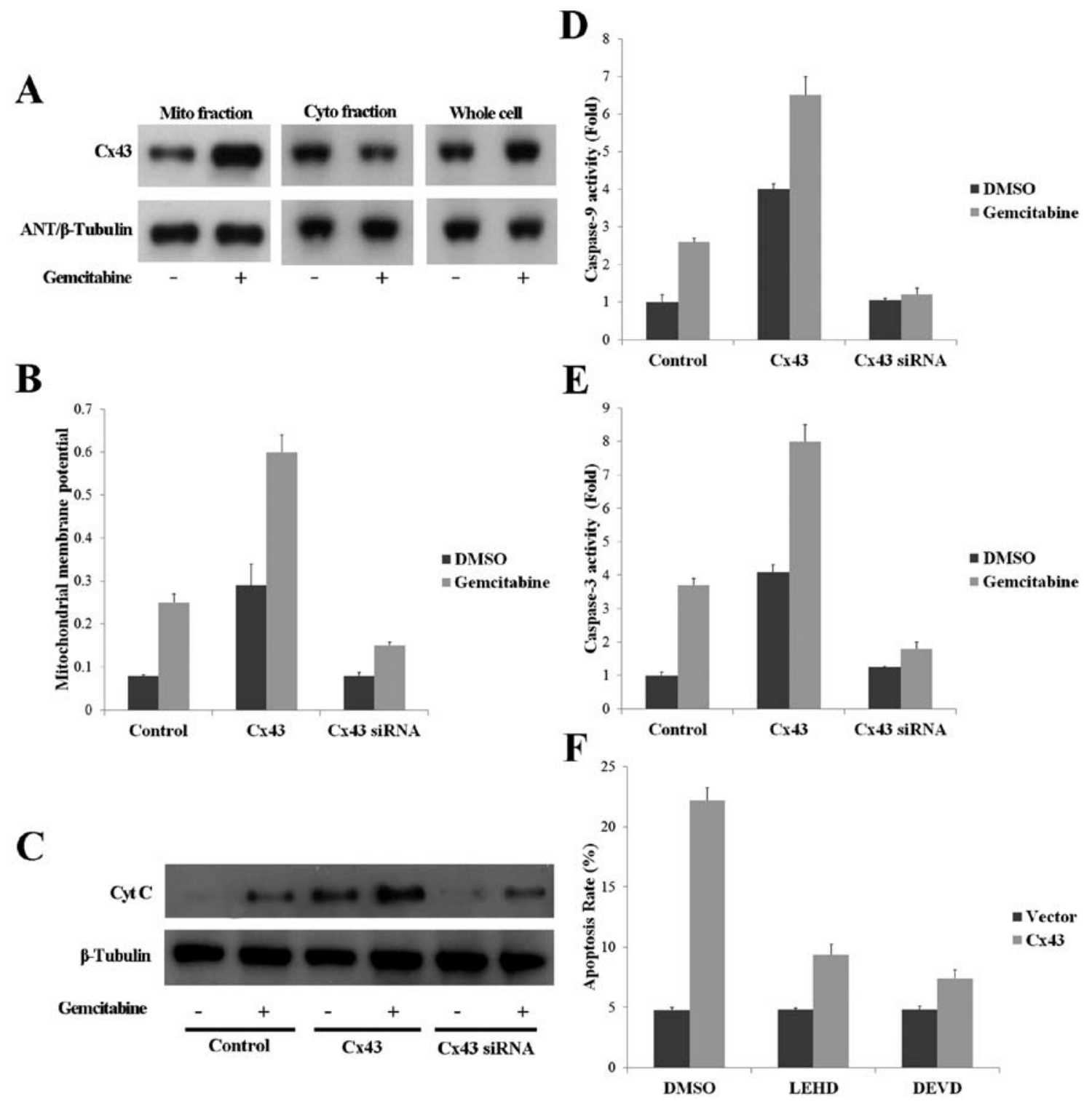

Figure 3. Cx43 regulates apoptosis via the mitochondrial apoptotic pathway. (A) Gemcitabine-induced Cx43 mitochondrial localization was analyzed by immunoblotting of mitochondrial fraction, cytosolic fraction and whole cell lysates. ANT was used as the loading control of mitochondrial fraction, and $\beta$-tublin for cytosolic and whole cell lysates. Mito fraction, miochondrial fraction; Cyto fraction, cytosolic fraction. (C) Release of Cyt c was assessed by western blot analysis of cytosolic fraction. After expression of $\mathrm{Cx} 43$ or knockdown of Cx43, the mitochondrial membrane potential (B), caspase-9 activity (D), caspase-3 activity (E) and apoptotic rate (F) were analyzed. Data represent the means \pm SEM values from at least 3 separate experiments.

cancer cell growth (Fig. 1E). As Cx43 is involved in regulating apoptosis in multiple cancers, we speculated that $\mathrm{Cx} 43$ may be a pro-apoptotic gene in pancreatic cancer. The apoptotic rates were approximately $20-30 \%$ when the pancreatic cancer cells were transfected with $\mathrm{Cx} 43$ (Fig. 1F). Gemcitabine is a widelyused drug for the first-line therapy of pancreatic cancer. In this study, we found that level of $\mathrm{Cx} 43$ positively correlated with the apoptosis induced by gemcitabine (Fig. 1G). These results suggest that $\mathrm{Cx} 43$ plays an important role in regulating the apoptosis of pancreatic cancer cells.

Tumor-suppressive function of CX43 occurs via a GJindependent pathway. It is well known that $\mathrm{Cx} 43$ exerts its function through a canonical GJ-mediated pathway. We therefore investigated whether GJ is necessary for $\mathrm{Cx} 43$ tumorsuppressive function. $18 \beta$-glycyrrhetinic acid ( $\beta$-GA) was used for GJ inhibition (Fig. 2A and B). With $\beta$-GA treatment, Cx43 still inhibited the growth and induced the apoptosis of BxPC-3 cells (Fig. 2C and D). As $\beta$-GA inhibits GJs formed by any Cx protein besides $\mathrm{Cx} 43$, the $\mathrm{Cx} 43$ mutant, $\mathrm{Cx} 43 \mathrm{~N}$, constructed by the deletion of amino acids 130-136 from the cytoplasmi loop, was used for inhibiting the Cx43-meditated GJ (8). GJ formation in $\mathrm{Cx} 43 \mathrm{~N}$-expressed BxPC-3 cells was dramatically decreased (Fig. 2A and B). However, the Cx43 mutant preserved tumorsuppressive function without forming GJs (Fig. 2C and D). These results suggest that a GJ is not the key mechanism involved in the anti-tumor effect of $\mathrm{Cx} 43$ in pancreatic cancer cells.

Cx43 induces apoptosis through mitochondrial apoptotic pathway. The idea of a GJ-independent pathway was further confirmed by $\mathrm{Cx} 43$ subcellular localization. While BxPC-3 cells were exposed to gemcitabine, $\mathrm{Cx} 43$ translocated from the 

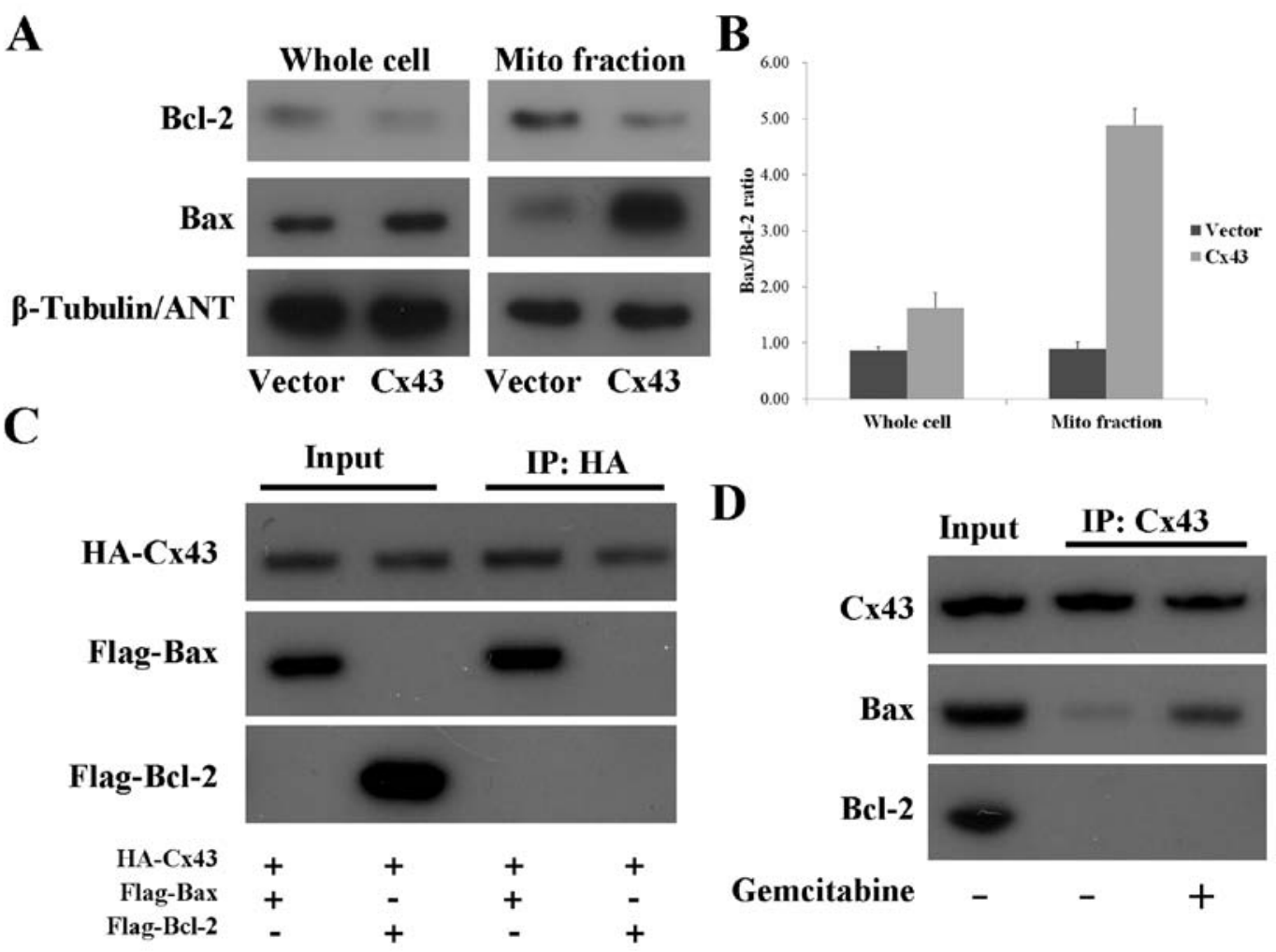

Figure 4. Cx43 interacts with Bax to regulate the apoptosis of pancreatic cancer cells. (A) Bax and Bcl-2 expression was assayed in both whole cell lysates and mitochondrial fractions. ANT was used as the loading control of mitochondrial fraction, and $\beta$-tubulin for whole cell lysates. (B) The Bax/Bcl-2 ratio was calculated by densitometric analysis of 3 separate experiments. The association of Cx43/Bax or Cx43/Bcl-2 was analyzed by co-immunoprecipitation. Mito fraction, miochondrial fraction (C) HA-Cx43 was immunoprecipitated by HA antibodies. Immunoblotting was performed with Flag antibody. (D) BxPC-3 cells were treated with or without gemcitabine. Endogenous Cx43 was immunoprecipitated by $\mathrm{Cx} 43$ antibodies. Then Bax or Bcl-2 antibodies were used for immunoblotting. IP, immunoprecipitation.

cytoplasm to the mitochondria (Fig. 2E). The characteristic of mitochondrial sublocalization was also assessed by cellular fractionation. Mitochondrial Cx43 expression was dramatically increased with gemcitabine treatment (Fig. 3A). We presumed that the $\mathrm{Cx} 43$ localization was related to the mitochondrial apoptotic pathway. The mitochondrial membrane potential (MMP) was increased when BxPC-3 cells were transfected with $\mathrm{Cx} 43$. The increase of MMP was more dramatic in the cells treated with gemcitabine simultaneously (Fig. 3B). The knockdown of Cx43 itself did not cause a significant change in MMP. However, the gemcitabine-induced decrease of MMP was compromised by the Cx43 knockdown (Fig. 3B). Another indicator of mitochondria-mediated apoptosis was the release of cytochrome c (Cyt c) (Fig. 3C). The activities of caspase-9 and caspase-3 were increased with $\mathrm{Cx} 43$ expression (Fig. 3D and E). When the cells were treated with the caspase inhibitors, LEHD (against caspase-9) and DEVD (against caspase-3), the Cx43-induced apoptosis was compromised (Fig. 3F). Overall, the mitochondrial apoptotic pathway was shown to be involved in the Cx43-induced apoptosis.

Cx43/Bax interaction is required to induce apoptosis of pancreatic cancer cells. Bcl-2 family proteins are master regulators of the mitochondrial apoptotic pathway. The Bax/Bcl-2 ratio serves as a rheostat to determine cell susceptibility to the intrinsic apoptotic signaling pathway. The expression of $\mathrm{Cx} 43$ in $\mathrm{BxPC}-3$ cells caused an increase in the Bax/Bcl-2 ratio (Fig. 4A and $\mathrm{B})$. Of note, the main change in $\mathrm{Bax}$ or $\mathrm{Bcl}-2$ was mitochondria-localized protein (Fig. 4A and B). As Cx43 translocated to the mitochondria during apoptosis, we speculated that potential interactions occurred between $\mathrm{Cx} 43$ and $\mathrm{Bcl}-2$ family proteins. BxPC-3 cells were co-transfected with HA-Cx43/Flag-Bax or HA-Cx43/Flag-Bcl-2. Co-immunoprecipitation revealed that Cx43 co-precipitated with Bax, but not with Bcl-2 (Fig. 4C). Reciprocal co-immunoprecipitation showed consistent results. As the above experiments relied on exogenous proteins, we sought to examine whether an interaction occurs between endogenous $\mathrm{Cx} 43$ and Bax proteins. We further examined the interaction in BxPC-3 cells treated with gemcitabine (Fig. 4D). There was no $\mathrm{Cx} 43 / \mathrm{Bcl}-2$ interaction to be found regardless of the overexpression of these proteins or apoptotic stimuli. On the contrary, gemcitabine increased the interaction between Cx43 and Bax (Fig. 4D). These findings suggest that $\mathrm{Cx} 43$ directly interacts with Bax, but not Bcl-2, to regulate the apoptosis of pancreatic cancer cells.

Cx43 (241-382aa) interacts with Bax to permeabilize mitochondrial membrane. We then examined the importance of the $\mathrm{Cx} 43 / \mathrm{Bax}$ interaction in regulating the apoptosis of pancreatic cancer cells. We examined the $\mathrm{Cx} 43$ protein to find the key region for its interaction with Bax. Cx43 includes 4 transmembrane regions and a connexin homolog domain (CNX). Different lengths of $\mathrm{Cx} 43$ plasmids were constructed as shown in Fig. 5A. Co-immunoprecipitation indicated that 241-382aa 
A
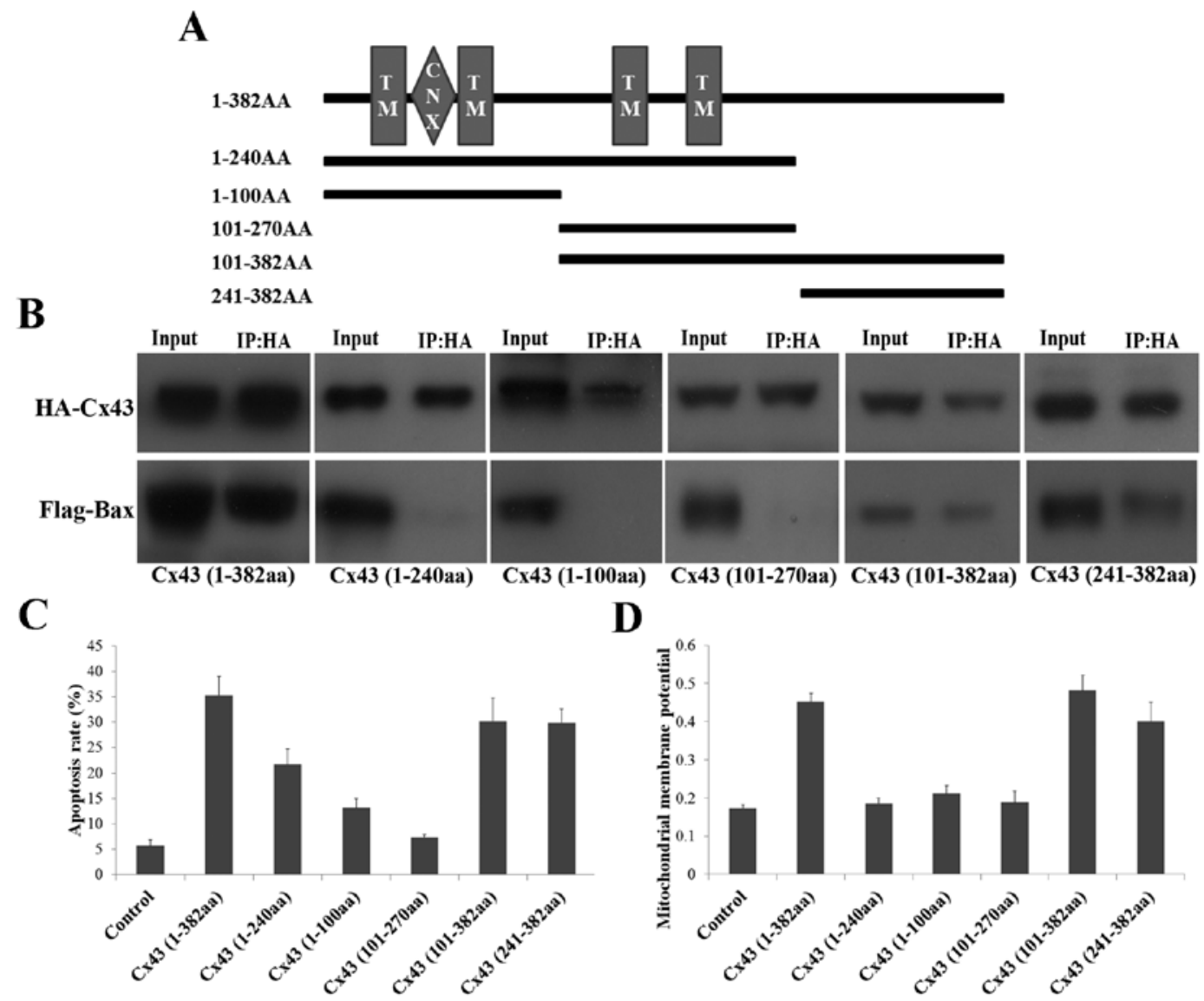

$\mathbf{E}$

$\mathbf{F}$
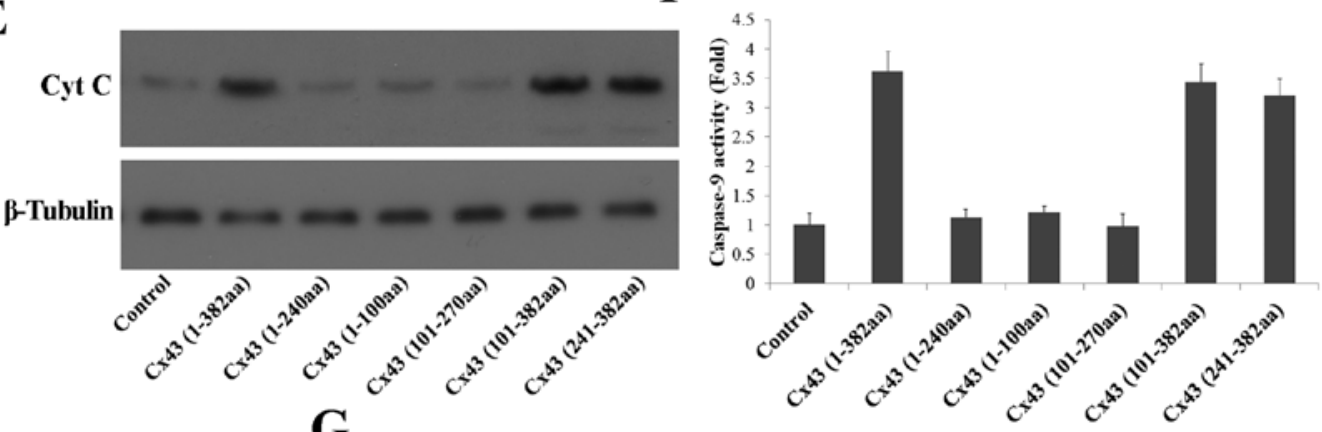

G

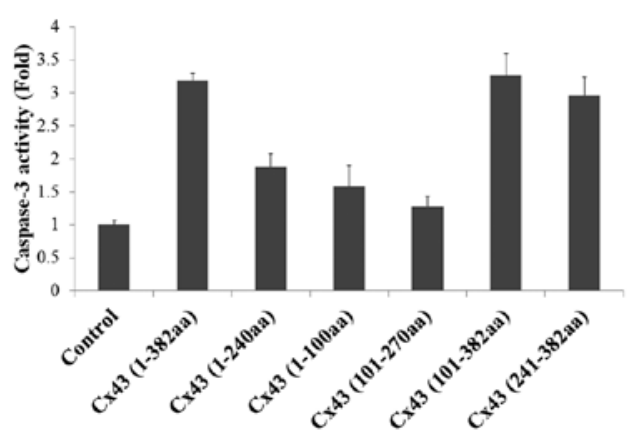

Figure 5. The 241-382aa fragment of $\mathrm{Cx} 43$ is required for initiating the mitochondrial apoptotic pathway. (A) As indicated, different lengths of the $\mathrm{Cx} 43$ plasmid were constructed with HA tag (TM, transmembrane domain; CNX, connexin homolog domain). (B) Cx43 was immunoprecipitated by HA antibody. Immunoblotting was performed with Flag antibody. IP, immunoprecipitation. After cells were transfected with different lengths of Cx43 plasmid or control plasmid, the apoptotic rate (C), mitochondrial membrane potential (D), caspase-9 activity (F) and caspase-3 activity (G) were analyzed. Data represent the means \pm SEM values from at least 3 separate experiments. Release of Cyt $\mathrm{c}$ was assessed by western blot analysis of cytosolic fraction, and $\beta$-tubulin was used as the loading control (E).

was required for the interaction with Bax (Fig. 5B). The Bax interacting region (241-382aa) induced apparent apoptosis of pancreatic cancer cells. Even though certain Cx43 plasmid lengths did not interact with Bax, there was still some impact on pancreatic cell apoptosis (Fig. 5C). However, the Bax interacting region (241-382aa) was required for depolarizing the 
mitochondrial membrane and releasing Cyt c (Fig. 5D and E). Caspase 9, as a mitochondria-mediated specific caspase, was activated by $\mathrm{Cx} 43$ fragments containing the 241-382aa region (Fig. 5F). Caspase-3, as a downstream effector caspase, was activated by either Bax interacting or non-interacting $\mathrm{Cx} 43$ fragments (Fig. 5G). These results indicate that the interaction between $\mathrm{Cx} 43$ and Bax is necessary for initiating the mitochondrial apoptotic pathway.

\section{Discussion}

$\mathrm{Cx} 43$ has been reported to exhibit anti-tumor effects in various types of cancer. The downregulation of $\mathrm{Cx} 43$ promotes an aggressive breast cancer cell phenotype (9). $\mathrm{Cx} 43$ is related to the occurrence, development and metastatic potential of gastric cancers (10). The tumor suppressive role of $\mathrm{Cx} 43$ has several aspects, including cell proliferation, invasion and metastasis. The expression of Cx43 in pancreatic cancer cells caused apparent growth inhibition. The subsequent rescue experiment further demonstrated that growth inhibition was regulated by Cx 43. Of note, pancreatic cancer cells showed an increase in the apoptotic rate with $\mathrm{Cx} 43$ expression. When the cells were treated with gemcitabine, which is the most widely used drug for pancreatic cancer, sensitivity to apoptosis correlated with the level of $\mathrm{Cx} 43$. Increased sensitivity to apoptotic stimuli was evident in the cells with higher levels of $\mathrm{Cx} 43$, such as CFPAC-1 cells. In the PANC-1 cells, the lower level of Cx43 rendered resistance to gemcitabine treatment. $\mathrm{Cx} 43$ has shown similar apoptotic regulation in other types of cancer. The overexpression of $\mathrm{Bcl}-2$ in $\mathrm{Cx} 43$-transfected cells confers resistance to apoptosis induced under low-serum conditions in human glioblastoma cells (11). Cx43 increases the sensitivity of prostate cancer cells to TNF $\alpha$-induced apoptosis (12). The protective effect of $\mathrm{Cx} 43$ has also been found in certain normal cells. In human epithelial cells, Cx43 has been shown to protect cells from oxidative stress (13). The different role of Cx43 is due to sophisticated mechanisms. Cx43 exerts its function through either GJ-dependent or -independent signaling pathways. Even in the case of GJs, it can transmit pro-apoptotic and anti-apoptotic signals to control the 'destiny' of cells.

We then tried to unravel the mechanism related to $\mathrm{Cx} 43$ regulated apoptosis. To differentiate between a GJ-dependent and -independent pathway, we used $\beta$-GA (a broad spectrum inhibitor of GJ) and a Cx43 mutant (a specific inhibitor of $\mathrm{Cx} 43$ mediated GJ). Even when GJs were significantly decreased, Cx43-regulated apoptosis was not compromised in pancreatic cancer cells. These data suggest that a GJ-dependent pathway is not required for $\mathrm{Cx} 43$-regulated apoptosis. GJ-independent pathway has attracted much attention in recent years (14). $\mathrm{Cx} 43$ controls $\mathrm{Ca}^{2+}$ homeostasis to regulate cell death induced by a variety of insults, which has no correlation with $\mathrm{Cx} 43$ mediated GJs (15). Increasing evidence has indicated that Cxs, including Cx43, may control cell growth and inhibit tumorigenicity independent of GJs $(14,16)$. The c-terminal tail of Cx43 is thought to be crucial for a GJ-independent pathway. Cx43 interacts with a large number of signaling proteins via intracellular carboxyl tail to regulate cellular functions $(17,18)$. From the results of the present study, it can be concluded that GJ-independent pathways play major roles in regulating the apoptosis of pancreatic cancer cells.
Our study showed that $\mathrm{Cx} 43$ translocated to the mitochondria with gemcitabine treatment. Other studies have also demonstrated that $\mathrm{Cx} 43$ is located at the mitochondria of cardiomyocytes besides the cell membrane $(19,20)$. In response to Wnt signaling, $\mathrm{Cx} 43$ interacts with $\beta$-catenin and translocates to the nucleus to regulate downstream gene expression (21). Further evidence has indicated that GJ-independent control of cell growth occurs through the aberrant localization of Cxs (14). The aberrant localization of Cxs leads to interactions with other signaling molecules or to the formation of hemichannels on organelles. Two major apoptotic pathways have been identified in mammalian cells: intrinsic and extrinsic pathways. The extrinsic apoptotic pathway is triggered by the engagement of so-called 'death receptors' on the cell surface. The intrinsic pathway is provoked by intercellular organelles, such as the mitochondria, endoplasmic reticulum and other organelles $(22,23)$. Our data showed that the intrinsic apoptotic pathway was activated by mitochondrial $\mathrm{Cx} 43$ in pancreatic cancer cells. MMP depolarization, the Cyt $\mathrm{c}$ release and specific caspase activation, characteristics of the mitochondrial apoptotic pathway, were induced by $\mathrm{Cx} 43$. Consistent with our results, other reports have shown that $\mathrm{Cx} 43$ is a key regulator of mitochondrial physiology and myocyte apoptosis $(19,24)$. Mitochondrial Cx43 is also a new player in the pathophysiology of myocardial ischemiareperfusion injury-related apoptosis $(19,25)$.

During stress, the Bcl-2 family is mainly responsible for the fate of cells (whether apoptosis will be induced or not) (26). Mitochondrial Bcl-2 family proteins are important to the intrinsic apoptotic pathway. The ratio of $\mathrm{Bax} / \mathrm{Bcl}-2$ was increased with $\mathrm{C} x 43$ expression. These results are consistent with those from a previous study on human glioblastoma cells by microarray analysis (27). Changes in Bax or Bcl-2 proteins were mainly related to mitochondrial localization. Co-immunoprecipitation assay showed that $\mathrm{Cx} 43$ interacted with Bax, instead of Bcl-2 to regulate the apoptosis of pancreatic cancer cells. It is possible that $\mathrm{Cx} 43$ interacts with other Bcl-2 family members. Further studies are required to elucidate potential interactions between $\mathrm{Cx} 43$ and other Bcl-2 family proteins.

To understand the importance of the $\mathrm{Cx} 43 / \mathrm{Bax}$ interaction in regulating cell apoptosis, different lengths of the $\mathrm{Cx} 43$ plasmid were constructed. Our data indicate that 241-382aa is required for the $\mathrm{Cx} 43 / \mathrm{Bax}$ interaction. This region is heavily modified by post-transcriptional regulation. The ERK (28), casein kinase 1 (29), c-Src (30) and PKC (31) phosphorylation sites reside in the 241-382aa region. These modifications are involved in regulating GJ and protein interactions. The 241-382aa fragment induced apparent apoptosis. While some $\mathrm{Cx} 43$ fragments, such as 1-240aa, did not interact with Bax, apoptosis still occurred. This suggested that other pathways were also involved, such as a GJ-dependent pathway. However, the Bax interacting region (241-382aa) was required for inducing the Cyt c release, MMP change and caspase- 9 activation. Therefore, the Cx43/Bax interaction was involved in prompting the mitochondrial apoptotic pathway. Therefore, subsequent mitochondrial membrane depolarization relies on Bax. Bax recruits Cx43 to the mitochondria, where $\mathrm{Cx} 43$ forms hemichannels to transduce apoptotic signals, including calcium signaling and $\mathrm{Cyt} \mathrm{c}$ release.

Taken together, our data indicate that $\mathrm{Cx} 43$ regulates the growth and apoptosis of pancreatic cancer cells. Cx43 interacts with Bax to initiate the mitochondrial apoptotic pathway. 


\section{Acknowledgements}

This study was supported by grants from the National Key Program for Basic Research of China (2010CB529902), the National Natural Science Foundation of China (81001008), and the Shanghai Leading Academic Discipline Project (S30205).

\section{References}

1. Dorado J, Lonardo E, Miranda-Lorenzo I and Heeschen C: Pancreatic cancer stem cells: new insights and perspectives. J Gastroenterol 46: 966-973, 2011.

2. Pluda JM and Parkinson DR: Clinical implications of tumorassociated neovascularization and current antiangiogenic strategies for the treatment of malignancies of pancreas. Cancer 78: 680-687, 1996

3. Neijssen J, Herberts C, Drijfhout JW, Reits E, Janssen L and Neefjes J: Cross-presentation by intercellular peptide transfer through gap junctions. Nature 434: 83-88, 2005.

4. Giepmans BN: Role of connexin43-interacting proteins at gap junctions. Adv Cardiol 42: 41-56, 2006.

5. Bui MM, Han G, Acs G, et al: Connexin 43 is a potential prognostic biomarker for ewing sarcoma/primitive neuroectodermal tumor. Sarcoma 2011: 971050, 2011.

6. Kalvelyte A, Imbrasaite A, Bukauskiene A, Verselis VK and Bukauskas FF: Connexins and apoptotic transformation. Biochem Pharmacol 66: 1661-1672, 2003.

7. Goldberg GS, Bechberger JF and Naus CC: A pre-loading method of evaluating gap junctional communication by fluorescent dye transfer. Biotechniques 18: 490-497, 1995.

8. Guan X, Wilson S, Schlender KK and Ruch RJ: Gap-junction disassembly and connexin 43 dephosphorylation induced by 18 beta-glycyrrhetinic acid. Mol Carcinog 16: 157-164, 1996.

9. Shao Q, Wang H, McLachlan E, Veitch GI and Laird DW: Downregulation of $\mathrm{Cx} 43$ by retroviral delivery of small interfering RNA promotes an aggressive breast cancer cell phenotype. Cancer Res 65: 2705-2711, 2005.

10. Wu J, Zhou HF, Wang CH, et al: Decreased expression of $\mathrm{Cx} 32$ and $\mathrm{Cx} 43$ and their function of gap junction intercellular communication in gastric cancer. Zhonghua Zhong Liu Za Zhi 29: 742-747, 2007 (In Chinese).

11. Huang R, Liu YG, Lin Y, et al: Enhanced apoptosis under low serum conditions in human glioblastoma cells by connexin 43 (Cx43). Mol Carcinog 32: 128-138, 2001.

12. Wang M, Berthoud VM and Beyer EC: Connexin43 increases the sensitivity of prostate cancer cells to TNFalpha-induced apoptosis. J Cell Sci 120: 320-329, 2007.

13. Hutnik CM, Pocrnich CE, Liu H, Laird DW and Shao Q: The protective effect of functional connexin 43 channels on a human epithelial cell line exposed to oxidative stress. Invest Ophthalmol Vis Sci 49: 800-806, 2008.

14. Cronier L, Crespin S, Strale PO, Defamie N and Mesnil M: Gap junctions and cancer: new functions for an old story. Antioxid Redox Signal 11: 323-338, 2009.
15. Garcia-Dorado D, Rodriguez-Sinovas A, Ruiz-Meana M, Inserte J, Agullo L and Cabestrero A: The end-effectors of preconditioning protection against myocardial cell death secondary to ischemiareperfusion. Cardiovasc Res 70: 274-285, 2006.

16. Jiang JX and Gu S: Gap junction- and hemichannel-independent actions of connexins. Biochim Biophys Acta 1711: 208-214, 2005.

17. Langlois S, Cowan KN, Shao Q, Cowan BJ and Laird DW: The tumor-suppressive function of Connexin43 in keratinocytes is mediated in part via interaction with caveolin-1. Cancer Res 70: 4222-4232, 2010.

18. Herrero-Gonzalez S, Gangoso E, Giaume C, Naus CC, Medina JM and Tabernero A: Connexin 43 inhibits the oncogenic activity of c-Src in C6 glioma cells. Oncogene 29: 5712-5723, 2010.

19. Goubaeva F, Mikami M, Giardina S, Ding B, Abe J and Yang J: Cardiac mitochondrial connexin 43 regulates apoptosis. Biochem Biophys Res Commun 352: 97-103, 2007.

20. Boengler K, Dodoni G, Rodriguez-Sinovas A, et al: Connexin 43 in cardiomyocyte mitochondria and its increase by ischemic preconditioning. Cardiovasc Res 67: 234-244, 2005.

21. Ai Z, Fischer A, Spray DC, Brown AM and Fishman GI: Wnt-1 regulation of connexin43 in cardiac myocytes. J Clin Invest 105: 161-171, 2000.

22. Ferri KF and Kroemer G: Mitochondria - the suicide organelles. Bioessays 23: 111-115, 2001.

23. Zhao X, Sun Y, Yu H, et al: Apoptosis induced by BIK was decreased with RNA interference of caspase-12. Biochem Biophys Res Commun 359: 896-901, 2007.

24. Penna C, Perrelli MG, Raimondo S, et al: Postconditioning induces an anti-apoptotic effect and preserves mitochondrial integrity in isolated rat hearts. Biochim Biophys Acta 1787: 794-801, 2009.

25. Ruiz-Meana M, Rodriguez-Sinovas A, Cabestrero A, Boengler K, Heusch G and Garcia-Dorado D: Mitochondrial connexin 43 as a new player in the pathophysiology of myocardial ischaemiareperfusion injury. Cardiovasc Res 77: 325-333, 2008.

26. Gustafsson AB and Gottlieb RA: Bcl-2 family members and apoptosis, taken to heart. Am J Physiol Cell Physiol 292: C45-C51, 2007.

27. Iacobas DA, Urban-Maldonado M, Iacobas S, Scemes E and Spray DC: Array analysis of gene expression in connexin-43 null astrocytes. Physiol Genomics 15: 177-190, 2003.

28. Cameron SJ, Malik S, Akaike M, et al: Regulation of epidermal grow th factor-induced connexin 43 gap junction communication by big mitogen-activated protein kinase1/ERK 5 but not ERK1/2 kinase activation. J Biol Chem 278: 18682-18688, 2003.

29. Cooper CD and Lampe PD: Casein kinase 1 regulates connexin-43 gap junction assembly. J Biol Chem 277: 44962-44968, 2002.

30. Lin R, Warn-Cramer BJ, Kurata WE and Lau AF: v-Src phosphorylation of connexin 43 on Tyr247 and Tyr265 disrupts gap junctional communication. J Cell Biol 154: 815-827, 2001.

31. Lampe PD, TenBroek EM, Burt JM, Kurata WE, Johnson RG and Lau AF: Phosphorylation of connexin 43 on serine 368 by protein kinase $\mathrm{C}$ regulates gap junctional communication. J Cell Biol 149: 1503-1512, 2000. 